УДК 159.9.019.4:364-78.24:636.082.454.5-055.2:316.812.1-055.52-055.6

Для цитирования: Штро Р.С., Гуткевич Е.В. Психологические риски вспомогательных репродуктивных технологий и особенности отношения родителей к детям, рождённым с помощью ВРТ (литературный обзор). Сибирский вестник психиатрии и наркологии. 2020; 2 (107): 51-59. https://doi.org/10.26617/1810-3111-20202(107)-51-59

\title{
Психологические риски вспомогательных репродуктивных технологий и особенности отношения родителей к детям, рождённым с помощью ВРТ (литературный обзор)
}

\section{1 Штро Р.C., 1, 2Гуткевич E.B.}

${ }^{I}$ Национальный исследовательский Томский государственный университет (НИ ТГУ) Россия, 634050, Томск, пр. Ленина, 36

${ }^{2}$ Научно-исследовательский институт психического здоровья, Томский национальный исследовательский медииинский центр Российской академии наук (НИИ психического здоровья Томский НИМЦ) Россия, 634014, Томск, ул. Алеутская, 4

\section{PEЗЮME}

Вспомогательные репродуктивные технологии (ВРТ) позволяют забеременеть, выносить и родить ребёнка людям с нарушениями репродуктивного здоровья. Зачатие, беременность и рождение ребёнка сопровождаются особыми психологическими условиями и рисками, а дальнейшее взаимодействие родителей и ребенка формирует вектор его психического развития. Фактами, обусловливающими актуальность темы, явились как наблюдаемая распространённость нарушений репродуктивного здоровья и рост использования вспомогательных репродуктивных технологий, так и показанные реальные психологические риски для родителей, использующих ВРТ, в частности экстракорпоральное оплодотворение (ЭКО), и их детей. Цель: по материалам отечественной и зарубежной литературы изучить психологические особенности применения вспомогательных репродуктивных технологий в семьях с бесплодием и систематизировать данные о наличии или отсутствии связей между их использованием и отношением родителей к детям, зачатым, выношенным и рождённым благодаря этим технологиям. Материал и методы: были рассмотрены статьи, опубликованные с 1990 по 2019 г. на русском и английском языках. Поиск производился с февраля по апрель 2020 г., с помощью онлайн-баз данных Google Scholar, eLibrary и Science Direct, предпочтение отдавалось полнотекстовым статьям последних лет, опубликованным в рецензируемых научных журналах. Поисковые запросы включали ключевые фразы: «психологические аспекты ВРТ/ЭКО», «детско-родительские отношения», «супружеские отношения» и другие, а также их аналоги на английском языке. В предыдущих исследованиях психологического благополучия родителей, использовавших вспомогательные репродуктивные технологии, редко рассматривались вопросы психологических рисков ВРТ для формирования детско-родительских отношений, в том числе специфика детско-отцовских отношений, а также психологических особенностей и динамики отношения родителей к детям в зависимости от возраста рождённого с помощью ЭКО ребенка. Изучение данной проблемы в будущем потребует дополнительных данных, касающихся в равной мере детско-материнских и детско-отцовских отношений, а также их динамики и внутренних особенностей различных сфер эмоционально-личностного общения родителей и детей разного возраста в семьях, использующих вспомогательные репродуктивные технологии.

Ключевые слова: психологические риски, бесплодие, вспомогательные репродуктивные технологии (ВРТ), экстракорпоральное оплодотворение (ЭКО), брачно-семейные отношения, психологические аспекты детско-родительских отношений.

\section{ВВЕДЕНИЕ}

Согласно данным статистического ежегодника, предоставляемого федеральной службой государственной статистики, в России в 2017 г. количество поставленных диагнозов бесплодия среди женщин составляло 857,4 случая на 100000 взрослого населения $(+1,71 \%$ по сравнению с 2016 г.), среди мужчин - 85,8 на 100000 взрослого населения (+7,11\% по сравнению с 2016 г.), соответственно в абсолютных числах - 284314 у женщин и 45385 случаев у мужчин [1]. Возможность людям с нарушениями репродуктивного здоровья забеременеть, выносить и успешно родить здорового ребёнка является целью вспомогательных репродуктивных технологий. Удешевление процедур ВРТ и рост информированности и образованности населения в вопросах репродуктивного здоровья стимулируют растущую популярность методов ВРТ. 
По состоянию на 2012 г. в мире официально было зарегистрировано 3,3-4,1 миллиона младенцев, рождённых с помощью ВРТ, а общее их число на тот момент оценивалось в 5 миллионов [2]; по данным на 18 февраля 2018 г. - в 7 миллионов [3]. К концу 2020 г. авторы прогнозируют цифры в 9-10 миллионов человек, а количество рождений превысит 500000 детей в год. Национальный регистр ВРТ в отчёте за 2017 г. оценивает общее количество циклов ВРТ, выполненных в 159 российских центрах ВРТ за этот год, в 139779 циклов (в 2016 г. 123181 цикл; +13,5\%) [4]. Эти данные являются основанием для подробного рассмотрения возможности существования психологических рисков и особенностей взаимоотношений для родителей и их детей в связи с бесплодием и применением вспомогательных репродуктивных технологий, вмешивающихся в естественный процесс зачатия.

\section{ЦЕЛЬ ИССЛЕДОВАНИЯ}

Изучить психологические особенности применения вспомогательных репродуктивных технологий в семьях с бесплодием и систематизировать данные о наличии или отсутствии связей между их использованием, в частности экстракорпорального оплодотворения, и отношением родителей (матерей и отцов) к детям, зачатым, выношенным и рождённым благодаря этим технологиям, по материалам отечественной и зарубежной литературы.

\section{МАТЕРИАЛЫ И МЕТОДЫ}

Материалом исследования явились научные публикации по теме работы, для этого был применен общенаучный метод поиска и анализа научной отечественной и зарубежной литературы по проблеме исследования, опубликованной за 29-летний период - с 1990 г. по 2019 г. на русском и английском языках в онлайнбазах данных научных публикаций Google Scholar, eLibrary и Science Direct. Поиск производился с февраля по апрель 2020 г. по ключевым запросам: «психологические аспекты ВРТ/ЭКО», «детско-родительские отношения», «супружеские отношения» и другие, а также их аналоги на английском языке. К первоначальному электронному поиску не применялись никакие критерии исключения; обобщение, сравнение и систематизация научнометодологических и экспериментальных данных в отношении психологических рисков применения вспомогательных репродуктивных технологий и психологических особенностей детско-родительских отношений в семьях, где дети рождены с их помощью.

\section{РЕЗУЛЬТАТЫ И ОБСУЖДЕНИЕ \\ Психологический риск при использова- нии вспомогательных репродуктивных тех- нологий}

Нередко при рассмотрении психологической ситуации применения вспомогательных репродуктивных технологий внимание уделяется положительным факторам психологического благополучия родителей и ребёнка.

Факт использования сложных, психотравмирующих и дорогостоящих ВРТ предполагает как высокий уровень стремления и готовности к материнству с преобладанием конструктивных мотивов беременности [5], так и ряд других защитных факторов, включающих более высокую удовлетворённость жилищными условиями и материальным положением [6], высокий социальный статус [7]. Также существует и обратная сторона психологической безопасности при применении ВРТ.

Фактором стрессорных воздействий в первую очередь является переживание нарушений репродуктивного здоровья, внутренняя картина болезни будущего родителя $[8,9,10]$. Фактически, помимо физиологических причин бесплодия, выделяются и психологические: депрессия, сексуальные страхи и фобии, чувство вины и сексуальные травмы в прошлом [11]. Эти факторы могут быть недооценены: в 30\% случаев бесплодия не удаётся обнаружить физиологические причины этих нарушений [12]. Спектр потенциальных психологических рисков не ограничен последствиями дистресса. Так, в ряде исследований показан значительный рост риска тревожности у женщин, использовавших ВРТ или проходящих лечение от бесплодия $[13,14]$. Фактором психологического риска в семьях, использующих ВРТ, является длительная история бесплодия $[15,16]$. В ряде работ показана связь длительного периода бесплодия у женщин участниц программ ЭКО с психологическими факторами: снижение самооценки, изменения в настроении и сексуальном функционировании [17], депрессия, гнев, тревога, социальная изоляция и чувство вины $[18,19]$. Мужчины с бесплодием сообщают о чувстве вины, стыда, низкой самооценке, гневе, изоляции, чувствах потери и личной неудачи [20, 21], тревожности и саморазрушении [22]. Как мужчины, так и женщины отмечают изменения в социальных контактах [23]. При этом возраст женщины, обратившейся за помощью в центры ВРТ, значительно выше среднего возраста женщин, забеременевших естественным путём [24]. 
Проведённые медицинские исследования показывают высокие риски соматических осложнений при беременности для женщин более старшего возраста [25]. С другой стороны, демонстрируют благотворное влияние беременности в старшем возрасте на постнатальное развитие ребёнка за счёт защитных факторов, таких как лучшие жилищные условия, материальное положение, высокий социальный статус матери, семьи [6].

Во-вторых, источником психологических рисков могут служить также особенности самой процедуры проведения ЭКО. В частности при беременности с использованием ВРТ наблюдаются повышенные риски соматических осложнений и репродуктивных потерь [24], что также вносит значительный вклад в психологическое благополучие родителей; например, на то, как женщины ощущают себя в роли матери [26].

Психологическое благополучие супругов и их взаимоотношений как условие успешности вспомогательных репродуктивных технологий

Данные о психологическом благополучии семей, использовавших вспомогательные репродуктивные технологии, далеко неоднозначны. В определённых случаях нарушения репродуктивного здоровья и бесплодие могут являться почти такими же интенсивными стрессогенными факторами, как потеря близкого, неизлечимая болезнь или прочие экстремально травмирующие ситуации [27]. Такие негативные переживания, связанные с диагностированным бесплодием и безуспешными попытками забеременеть естественным путём, могут оказывать неблагоприятное влияние на психологическое благополучие супругов и их брачно-семейные взаимоотношения [28]. Ситуация бесплодия и применение медицинских процедур могут являться стрессогенными факторами, повышающими напряжение в отношениях между супругами [29] и осложняющими коммуникацию между ними [30].

С другой стороны, в лонгитюдных исследованиях указывается, что само качество супружеских отношений в семье влияет как на индивидуальное здоровье и благополучие супружеской пары, вызывая депрессивную симптоматику [31], так и на переживание родительства [32]. Недостаток взаимопонимания между супругами закономерно может повлечь за собой отчуждение, депрессию, множественные психологические и соматические нарушения здо- ровья [29]. Психологическое напряжение в семье, депрессивная симптоматика, стрессовые ситуации, тревожность и иные неблагоприятные факторы могут стать психологической причиной нарушений репродуктивного здоровья и возникновения бесплодия [33, 34, 35, 36], и, как следствие, повлечь за собой использование вспомогательных репродуктивных технологий.

Эмпирические данные о психологическом благополучии супругов, использовавших вспомогательные репродуктивные технологии, противоречивы. Так, в работах 1996 г. и 2012 г. не было обнаружено значимых различий в статистике разводов в группах естественной беременности и ВРТ [37, 38]. В опубликованной в 2018 г. работе T.G. Bokhan et al. не удалось обнаружить связи между психологическими состояниями, стрессом и качеством семейных отношений в семьях с использованием ЭКО и естественной беременностью [39]. В ряде других исследований показана разница в удовлетворённости отношениями между группами с естественной и индуцированной беременностью. В семьях, использовавших ВРТ, удовлетворённость отношениями у супругов ниже, чем в контрольной группе, в которой беременность наступала естественным путём [37, 40]. Авторами подчеркиваются значительные сложности в освоении собственной гендерной роли у женщин [26].

Наблюдаемые эмпирические свидетельства не позволяют сделать однозначное заключение о влиянии вспомогательных репродуктивных технологий на психологическое благополучие супругов и их эмоционально-личностное общение. В исследовании А.P. ДроздиковойЗариповой (2011) было показано увеличение уровня психоэмоциональных нарушений в зависимости от длительности лечения и его сложности [41]. Вероятно, с возрастанием сложности ситуации и количества психотравмирующих факторов, риск психоэмоциональных нарушений растёт нелинейно. В таком случае при относительно спокойной и безопасной ситуации использования ВРТ у будущих родителей имеется достаточно психологических ресурсов, чтобы справляться с трудностями на том же уровне, что и в семьях с естественной беременностью. Однако при снижении психологической безопасности ситуации супругам будет гораздо труднее справиться с психоэмоциональным напряжением, не прибегая к помощи специалиста. 


\section{Эмоциональное и личностное общение родителей и ребёнка в семьях с ВРТ}

Проблематика различий в отношении к детям в семьях с разными видами беременности (естественной и беременностью, достигнутой с помощью ВРТ), мало изучена. В данной статье уже обсуждался факт возрастных различий между женщинами, забеременевшими естественным путём, и участницами программ ВРТ.

Исходя из этого, оценка психологических рисков применения вспомогательных репродуктивных технологий часто основывается на сравнительных результатах исследований матерей различных возрастов $[42,43,44,45]$.

Помимо этого, многие работы посвящены проблемам изучения детско-материнских отношений, их особенностей и формирования, в то время как специфика детско-отцовских отношений и динамика их развития часто остаётся за пределами исследовательских интересов [46]. Фундамент формирования отношения матери к ребёнку закладывается ещё во время беременности. В соответствии с данными, приводимыми McMahon et al., матери-участницы программ ВРТ ещё в период беременности испытывают повышенную тревогу по отношению к будущему ребёнку, воспринимают его как более уязвимого [47]. Это также может являться следствием связей между тревогой и возрастом матери. Так, в работе Spillman (1999) указывается на прямую зависимость силы тревоги за будущего ребёнка от возраста матери [48]. Уже после родов матери-участницы процедур ВРТ показывают более высокий уровень эмоциональной вовлечённости во взаимодействие с новорожденным [49].

В более старшем возрасте, вероятно, различия нивелируются. Так, в работе Colpin et al. (1995) показано, что уровень эмоциональной поддержки матерей по отношению к двухлетним детям не имел статистических значимых различий между женщинами-участницами программ ВРТ и контрольной группой женщин с естественным зачатием [50]. В исследовании Hahn и Di Pietro (2001) были проанализированы самоотчёты матерей относительно выражения эмоций при взаимодействии с их 3-7-летними детьми. Согласно результатам исследования не выявлено статистически значимых различий в эмоциональном взаимодействии матерей и их детей, однако учителя этих детей оценивали матерей из группы ЭКО как демонстрирующих большую теплоту, но не гиперопеку. По итогам интервью отмечались более низкие оценки агрессивного поведения родителей по отношению к своим детям. Учителя также оценили детей из семей с ЭКО как имеющих меньшее количество поведенческих проблем, чем у детей контрольной группы. Отдельное внимание в работе авторами уделено тому факту, что учителя не владели информацией о том, к какой из групп зачатия ребенка относилась та или иная семья [51].

В исследованиях на российских выборках также было показано, что качество жизни и благополучие семей, прибегавших к ВРТ, было выше, чем в контрольной группе [52]. В работе Е.В. Соловьевой был выделен ряд общих признаков, в равной степени характерных как для матерей-участниц программ ЭКО, так и участниц из контрольной группы. К ним был отнесён уровень положительных эмоций, который матери испытали при первом контакте с новорожденным, а также радость от индивидуальных особенностей ребёнка и беспокойство за его здоровье и развитие [53]. В этом же исследовании были описаны различия между группами женщин в отношении к своему ребёнку. Так, матери из группы ЭКО чаще отмечали негативное впечатление от первой встречи с ребёнком, чаще связывали своё беспокойство с поведением ребёнка, а также имели завышенные ожидания относительно способностей ребёнка.

Психологическая ситуация использования вспомогательных репродуктивных технологий оказывает влияние на родительскую идентичность и отношение супругов к собственному ребёнку на разных этапах (стадиях) его развития. На данный момент специфика этого воздействия, особенно относительно детскоотцовских отношений, недостаточно изучена. Основная часть опубликованных работ сконцентрирована на изучении детско-родительских отношений в период беременности и первых месяцев жизни ребёнка, в то время как развитие ребёнка во взаимосвязи с родителями после первого года жизни рассматривается лишь в небольшом количестве публикаций.

\section{ЗАКЛЮЧЕНИЕ}

Результаты аналитического обзора научной литературы показали, что зачатие, вынашивание и рождение ребёнка сопровождаются особыми психологическими условиями, которым необходимо уделять внимание при рассмотрении возможных психологических рисков использования вспомогательных репродуктивных технологий. 
Фактами, обусловливающими актуальность тематики, являются наблюдаемая распространённость нарушений репродуктивного здоровья и повышение частоты использования вспомогательных репродуктивных технологий. В обсуждаемых в обзоре исследованиях отражены реальные психологические риски для родителей (как матерей, так и отцов) в семьях, использующих BPT, например, такие как психоэмоциональные переживания нарушений репродуктивного здоровья и внутренняя картина болезни, психолого-сексуальные проблемы, тревожность и страхи, связанные с процедурой ЭКО. На данный момент не было найдено лонгитюдных исследований, в которых бы проводилась сравнительная оценка динамики стрессовых состояний и уровня тревоги у родителей, зачавших ребёнка как естественным путём, так и у использовавших ВРТ.

Данные о психологическом (физическом, психическом, социальном, эмоциональном) благополучии семей, использовавших вспомогательные репродуктивные технологии, неоднозначны и спорны. В более ранних работах не было обнаружено значимых различий в статистике разводов в группах естественной беременности и ВРТ. Хотя позднее отмечалось, что ситуация бесплодия и применение медицинских процедур могут выступать стрессогенными факторами, повышающими напряженность в отношениях между супругами и осложняющими коммуникативное взаимодействие между ними; отмечены значительные сложности в освоении собственной гендерной роли у женщин. Вероятно, с возрастанием сложности ситуации и количества психотравмирующих факторов риск психоэмоциональных нарушений растёт нелинейно.

Проблематика различий в отношении к детям в семьях с естественной беременностью и беременностью с помощью ВРТ изучена не в полной мере. В исследованиях на российских выборках обнаружено, что качество жизни и благополучие семей, прибегавших к ВРТ, было выше. В зарубежных исследованиях показано, что матери, использовавшие вспомогательные репродуктивные технологии, гораздо более тревожно относятся к будущему ребёнку на этапе беременности и в первые месяцы его жизни. Однако со временем последствия тревоги у матерей в группе ЭКО сравниваются с таковыми показателями матерей, зачавших и родивших своих детей естественным путём, без применения процедур ВРТ.
Результаты проведенных исследований позволяют оценить возрастной промежуток ребёнка, на котором происходит «выравнивание» показателей, как первую половину периода раннего детства - приблизительно от 1 года до 2 лет. При этом специфика детско-отцовских отношений и динамика их развития часто остаются за пределами исследовательских интересов.

Дальнейшее изучение проблемы требует дополнительных данных, касающихся в равной мере детско-материнских и детско-отцовских отношений, а также их динамики и внутренних особенностей различных сфер эмоциональноличностного общения родителей и ребёнка более старшего возраста, чем младенческий.

\section{КОНФЛИКТ ИНТЕРЕСОВ}

Авторы заявляют об отсутствии явных и потенциальных конфликтов интересов в связи с публикацией данной статьи.

\section{ИСТОЧНИК ФИНАНСИРОВАНИЯ}

Авторы декларируют об отсутствии финансирования или спонсорской поддержки при проведении исследования.

\section{ЛИТЕРАТУРA/REFERENCES}

1. Статистический сборник 2017 год / Общая заболеваемость взрослого населения России в 2017 году. Министерство здравоохранения Российской Федерации [Электронный pecypc]. Statistical digest 2017 / General incidence of the adult population of Russia in 2017. Ministry of Health of the Russian Federation [Electronic resource] (in Russian). URL: https://www.rosminzdrav.ru/ministry/61/22/stranitsa979/statisticheskie-i-informatsionnyematerialy/statisticheskiy-sbornik-2017-god

2. Adamson G.D., Tabangin M., Macaluso M., Mouzon $\mathrm{J}$. The number of babies born globally after treatment with the assisted reproductive technologies (ART). Fertility and Sterility. 2013; 100(3): S42. DOI: 10.1016/j.fertnstert.2013.07.1807

3. ART fact sheet. European Society of Human Reproduction and Embryology [Электронный ресурс]. URL: https://www.eshre.eu/ /media/sitecorefiles/Guidelines/ART-fact-sheet_vFebr18_VG.pdf

4. ВРТ в России. Отчет за 2017 год. Российская Ассоциация Репродукции Человека [Электронный pecypc]. ART in Russia. Report for 2017. Russian Association of Human Reproduction [Electronic resource] (in Russian). URL: http://www.rahr.ru/d_registr_otchet/RegistrART2017.pdf

5. Маленова А.Ю., Кытькова И. Г. Отношение женщин в ситуации ЭКО к беременности, ребенку, материнству. Педиатр. 2015; 6(4): 97 104. Malenova A.Yu., Kytkova I. G. The attitude of women in the IVF situation towards pregnancy, the child, motherhood. Pediatrician. 2015; 6 (4): 97-104 (in Russian). DOI: 10.17816/PED6497-104 
6. Воронина И.Д., Бохан Т.Г., Терехина О.В., Уайт Э.К., Малых С.Б., Ковас Ю.В. Демографические показатели, образ жизни и здоровье в семьях с естественной и индуцированной беременностью в России и Великобритании. Теоретическая и экспериментальная психология. 2016; 9(4): 63-76. Voronina I.D., Bokhan T.G., Terekhina O.V., White E.K., Malykh S.B., Kovas Yu.V. Demographics, lifestyle and health in families with natural and induced pregnancy in Russia and the UK. Theoretical and Experimental Psychology. 2016; 9 (4): 63-76 (in Russian).

7. Chen T.-H., Chang S.-P., Tsai C.-F., Juang K.-D. Prevalence of depressive and anxiety disorders in an assisted reproductive technique clinic. Human Reproduction. 2004; 19(10): 2313-2318. DOI: $10.1093 /$ humrep/deh414

8. Гуткевич Е. В., Шатунова А. И. Межпоколенная передача психической травмы, психогенеалогия и психическое здоровье в семье (феноменологическое исследование). Сибирский вестник психиатрии и наркологии. 2019; 3 (104): 21-32. Gutkevich E.V., Shatunova A.I. Intergenerational transmission of mental trauma, psychogenesis and mental health in the family (phenomenological study). Siberian Herald of Psychiatry and Addiction Psychiatry. 2019; 3 (104): 21-32 (in Russian). DOI: 10.26617/1810-3111-2019-3(104)-21-32

9. Куприянова И.Е., Чуйкова К.И., Ковалева Т.А., Спивак С.В., Петрова Е.И., Минакова Ю.В. Психотерапевтические подходы в комплексной реабилитации беременных с хроническими гепатитами В и С. Сибирский вестник психиатрии и наркологии. 2017; 1 (94): 53-56. Kupriyanova I.E., Chuikova K.I., Kovaleva T.A., Spivak S.V., Petrova E.I., Minakova Yu.V. Psychotherapeutic approaches in the course of complex rehabilitation of pregnant women with chronic hepatitis B and C. Siberian Herald of Psychiatry and Addiction Psychiatry. 2017; 1 (94): 53-56 (in Russian).

10. Ротова И.А., Куприянова И.Е., Ротова Н.А. Способ психотерапии женского бесплодия. Патент на изобретение RU $2687565 \mathrm{C} 1$, A61M 21/00(2006.01), 15.05.2019. Заявка № 2018104110 от 01.02.2018. Rotova I.A., Kupriyanova I.E., Rotova N.A. The method of psychotherapy of female infertility. Patent for invention RU $2687565 \mathrm{C} 1$, A61M 21/00(2006.01), 05/15/2019. Application no. 2018104110 dated 02/01/2018 (in Russian).

11. Michetti P.M., Rossi R., Bonanno D., Tiesi A., Simonelli C. Male sexuality and regulation of emotions: a study on the association between alexithymia and erectile dysfunction (ED). International Journal of Impotence Research. 2006; 18(2): 170174. DOI: $10.1038 /$ sj.ijir.3901386

12. Ray A., Shah A., Gudi A., Homburg R. Unexplained infertility: an update and review of practice. Reproductive Biomedicine Online. 2012; 24(6): 591-602. DOI: 10.1016/j.rbmo.2012.02.021
13. Gourounti K., Anagnostopoulos F., Potamianos G., Lykeridou K., Schmidt L., Vaslamatzis G. Perception of control, coping and psychological stress of infertile women undergoing IVF. Reproductive Biomedicine Online. 2012; 24(6): 670-679. DOI: 10.1016/j.rbmo.2012.03.002

14. Hjelmstedt A., Widström A.M., Wramsby H., Matthiesen A.S., Collins A. Personality factors and emotional responses to pregnancy among IVF couples in early pregnancy: a comparative study. Acta Obstet Gynecol Scand. 2003; 82(2): 152-161. DOI: $10.1034 / \mathrm{j} .1600-0412.2003 .00040 . \mathrm{x}$

15. Eriksen K. Infertility and the search for family. The Family Journal. 2001; 9(1): 55-61. DOI: $10.1177 / 1066480701091013$

16. Lee G.L., Blyth E.D., Chan C.L.W. Understanding the patterns of adjustment to infertility of IVF users using narrative and autobiographical timeline. Asian Pacific Journal of Reproduction. 2012; 1(2): 125-134. DOI: 10.1016/S2305-0500(13)60063-9

17. Downey J., McKinney M. The psychiatric status of women presenting for infertility evaluation. American Journal of Orthopsychiatry. 1992; 62(2): 196205. DOI: $10.1037 / \mathrm{h} 0079335$

18. Clayton A.H. Mental health concerns with infertility. Primary Psychiatry. 2004 May; 11(5): 17-18.

19. Schmidt L. Psychosocial burden of infertility and assisted reproduction. Lancet. 2006; 367(9508): 379-380. DOI: 10.1016/S0140-6736(06)68117-8

20. Myers M.F. Male gender-related issues in reproductive technology. Washington, DC: American Psychiatric Association Publishing, 1990: 25-35.

21. Wright J., Duchesne C., Sabourin S., Bissonnette F., Benoit J., Girard Y. Psychosocial distress and infertility: men and women respond differently. Fertility and Sterility. 1991; 55(1): 100-108. DOI: 10.1016/S0015-0282(16)54067-9

22. Glover L., Gannon K., Sherr L., Abel P. D. Distress in sub-fertile men: a longitudinal study. Journal of Reproductive and Infant Psychology. 1996; 14(1): 23-36. DOI: 10.1080/02646839608405856

23. Peterson B.D., Gold L., Feingold T. The experience and influence of infertility: Considerations for couple counselors. The Family Journal. 2007; 15(3): 251-257. DOI: 10.1177/1066480707301365

24. Якупова В.А. Особенности становления внутренней позиции матери при использовании вспомогательной репродуктивной технологии ЭКО: автореф. дис. ... к.м.н. М., 2015; 37. Үаkupova V.A. Features of the formation of the internal position of the mother when using assisted reproductive technology IVF: PhD thesis. Moscow, 2015; 37 (in Russian).

25. Kenny L.C., Lavender T., McNamee R., O’Neill S.M., Mills T., Khashan A.S. Advanced maternal age and adverse pregnancy outcome: evidence from a large contemporary cohort. Plos One. 2013; 8(2): 1-9. DOI: 10.1371/journal.pone.0056583 
26. Себелева Ю.Г. Гендерная идентичность у женщин, страдающих бесплодием, в программе экстракорпорального оплодотворения : автореферат дис. ... к.п.н. М., 2014: 26. Sebeleva Yu.G. Gender identity in women with infertility, in vitro fertilization program: PhD thesis. Moscow, 2014; 26 (in Russian).

27. Наку Е.А., Ковас Ю.В., Бохан Т.Г., Терехина О.В., Видякина Т.А. Факторы негативных психоэмоциональных состояний женщин, проходящих лечение бесплодия по программе ЭКО. Сибирский психологический журнал. 2017; 63: 119-135. Naku E.A., Kovas Yu.V., Bokhan T.G., Terekhina O.V., Vidyakina T.A. Factors of negative psycho-emotional states of women undergoing IVF treatment. Siberian Psychological Journal. 2017; 63: 119-135.DOI: 10.17223/17267080/63/9

28. de Faria D.E., Grieco Ch.S., de Barros S.M. The effects of infertility on the spouses' relationship. Revista da Escola de Enfermagem da USP. 2012 Aug; 46(4): 794-801. DOI: 10.1590/s008062342012000400002

29. Greil A.L., Slauson-Blevins K., McQuillan J. The experience of infertility: a review of recent literature. Sociology of Health \& Illness. 2010; 32(1): 140-162. DOI: 10.1111/j.1467-9566.2009.01213.x

30. Schmidt L., Holstein B.E., Christensen U., Boivin J. Communication and coping as predictors of fertility problem stress: cohort study of 816 participants who did not achieve a delivery after 12 months of fertility treatment. Human Reproduction. 2005; 20(11): 32483256. DOI: 10.1093/humrep/dei193.

31. Vujeva H.M., Furman W. Depressive symptoms and romantic relationship qualities from adolescence through emerging adulthood: A longitudinal examination of influences. Journal of Clinical Child \& Adolescent Psychology. 2011; 40(1): 123135. DOI: 10.1080/15374416.2011.533414

32. Kouros C.D., Papp L.M., Cummings E.M. Interrelations and moderators of longitudinal links between marital satisfaction and depressive symptoms among couples in established relationships. Journal of Family Psychology. 2008; 22(5): 667. DOI: 10.1037/0893-3200.22.5.667

33. Гарганеева А.Э., Востриков В.В., Фадеева Н.И. Эмоциональное состояние и социальные особенности пациенток в результативных и нерезультативных циклах экстракорпорального оплодотворения. Неврологический вестник (Казань). 2011; 43(2): 27-31. Garganeeva A.E., Vostrikov V.V., Fadeeva N.I. Emotional state and social peculiarities of women during successful and unsuccessful in-vitro fertilization cycles. Neurological Bulletin (Kazan). 2011; 43 (2): 27-31 (in Russian).

34. Ebbesen S.M.S., Zachariae R., Mehlsen M.Y., Thomsen D., Højgaard A., Ottosen L., Petersen T., Ingerslev H.J. Stressful life events are associated with a poor in-vitro fertilization (IVF) outcome: a prospective study. Human Reproduction. 2009; 24(9: 2173-2182. DOI: 10.1093/humrep/dep185
35. Frederiksen Y., Farver-Vestergaard I., Skovgård N.G., Ingerslev H.J., Zachariae R. Efficacy of psychosocial interventions for psychological and pregnancy outcomes in infertile women and men: a systematic review and meta-analysis. BMJ Open. 2015; 5(1): e006592. DOI: 10.1136/bmjopen-2014-006592

36. Galhardo A., Cunha M., Pinto-Gouveia J. Psychological aspects in couples with infertility. Sexologies. 2011; 20(4): 224-228. DOI: 10.1016/j.sexol.2011.08.005

37. Blake L., Casey P., Jadva V., Golombok S. Marital stability and quality in families created by assisted reproduction techniques: a follow-up study. Reproductive Biomedicine Online. 2012; 25(7): 678-683. DOI: 10.1016/j.rbmo.2012.09.006

38. Golombok S., Brewaeys A., Cook R., Giavazzi M.T., Guerra D., Mantovani A., Hall E., Crosignani P.G., Dexeus S. Children: the European study of assisted reproduction families: family functioning and child development. Human Reproduction. 1996; 11(10): 2324-2331. DOI: 10.1093/oxfordjournals.humrep.a019098

39. Bokhan T.G., Terekhina O.V., Shabalovskaya M.V., Leshchinskaia S.B., Silaeva A.V., Naku E.A., Selita F., Agarkova L.A. Spouses' psychological states and family Relations in families with natural and induced Pregnancies. Psychology in Russia: State of the art. 2018; 11(4): 50-67. DOI: 10.11621/pir.2018.040

40. Salvatore P., Gariboldi S., Offidani A., Coppola F., Amore M., Maggini C. Psychopathology, personality, and marital relationship in patients undergoing in vitro fertilization procedures. Fertility and Sterility. 2001; 75(6): 1119-1125. DOI: 10.1016/S00150282(01)01775-7

41. Дроздикова-Зарипова А.Р. Психоэмоциональные состояния женщин, получающих помощь по программе вспомогательных репродуктивных технологий. Международныцй журнал прикладных и фундаментальньхх исследований. 2011; 6-1: 7779. Drozdikova-Zaripova A.R. Psycho-emotional conditions of women receiving assistance under the program of assisted reproductive technologies. International Journal of Applied and Basic Research. 2011; 6-1: 77-79 (in Russian).

42. Гарданова Ж.Р. Пограничные психические расстройства у женщин с бесплодием в программе экстракорпорального оплодотворения и их психотерапевтическая коррекция : автореферат дис. ... д.м.н. M., 2007: 34. Gardanova J.R. Borderline mental disorders in women with infertility in the program of in vitro fertilization and their psychotherapeutic correction: abstract of MD thesis. Moscow, 2007: 34 (in Russian).

43. Захарова Е.И., Торчинова Ю.А. Условия становления материнской идентичности. Известия Пензенского государственного педагогического университета им. В.Г. Белинского. 2012; 28: 1234-1239. Zakharova E.I., Torchinova Yu.A. Conditions for the formation of maternal identity. Bulletin of the Penza State Pedagogical University named after V.G. Belinsky. 2012; 28: 1234-1239 (in Russian). 
44. Захарова Е.И. Особенности взаимодействия матерей с детьми в условиях «позднего» материнства. Национальный психологический журнал. 2014; 2 (14): 95-99. Zakharova E.I. Features of the interaction of mothers with children in the conditions of "late" motherhood. National Psychological Journal. 2014; 2 (14): 95-99 (in Russian).

45. Aasheim V., Waldenström U., Rasmussen S., Schytt E. Experience of childbirth in first-time mothers of advanced age-a Norwegian populationbased study. BMC Pregnancy and Childbirth. 2013; 13(1): 53. DOI: 10.1186/1471-2393-13-53

46. Авдеева Н.Н. Раннее вмешательство и эмоционально-личностные нарушения в раннем возрасте. Психолого-педагогические исследования. 2010; 2(5): 140-147. Avdeeva N.N. Early intervention and emotional-personality disturbances at an early age. Psychological and Pedagogical Research. 2010; 2 (5): 140-147 (in Russian).

47. McMahon C.A., Ungerer J.A., Beaurepaire J., Tennant C., Saunders D. Anxiety during pregnancy and fetal attachment after in-vitro fertilization conception. Human Reproduction (Oxford, England). 1997; 12(1): 176-182. DOI: 10.1093/humrep/12.1.176

48. Spillman J.A. Antenatal and postnatal influences on family relationships. London and New York: Routledge; 1999: 19-35.

49. Van Balen F. Child-rearing following in vitro fertilization. Journal of Child Psychology and Psychiatry. 1996; 37(6): 687-693. DOI: 10.1111/j.14697610.1996.tb01460.x

50. Colpin H., Demyttenaere K., Vandemeulebroecke L. New reproductive technology and the family: The parent child relationship following in vitro fertilization. Journal of Child Psychology and Psychi- atry. 1995; 36(8): 1429-1441. DOI: 10.1111/j.14697610.1995.tb01673.x

51. Hahn C.S., DiPietro J. A. In vitro fertilization and the family: Quality of parenting, family functioning, and child psychosocial adjustment. Developmental Psychology. 2001; 37(1: 37. DOI: 10.1037/0012-1649.37.1.37

52. Ланцбург М.Е., Соловьева Е.В. Сравнительное исследование материнского отношения к детям раннего возраста, зачатым при помощи ЭКО и спонтанно. У истоков развития. Сборник тезисов участников Четвертой Всероссийской научно-практической конференции по психологии развития. М.: ГБОУ МГППУ, 2013: 41-43. Lanzburg M.E., Solovieva E.V. A comparative study of maternal attitudes towards young children conceived using IVF and spontaneously. At the origins of development. The Book of Abstracts of participants of the Fourth All-Russian scientificpractical conference on developmental psychology. Moscow: Moscow State University of Psychology and Education, 2013: 41-43 (in Russian).

53. Соловьева Е.В. О материнском отношении к детям раннего возраста, зачатым посредством экстракорпорального оплодотворения. Психолого-педагогические исследования. 2014; 6(4): 147156. Solovieva E.V. On the maternal attitude towards young children conceived through in vitro fertilization. Psychological and Pedagogical Research. 2014; 6(4): 147-156 (in Russian).

Поступила в редакцию 20.04.2020 Утверждена к печати 01.06.2020

\section{Штро Роман Сергеевич - студент факультета психологии НИ ТГУ.}

Гуткевич Елена Владимировна - д-р мед. наук, ведущий научный сотрудник отделения эндогенных расстройств. Researcher ID O-1311-2014. Author ID Scopus 7801397871. ORCID 0000-0001-7416-7784. Author ID РИНЦ 165414. SPIN-код РИНЦ 6427-9007.

\section{Гуткевич Елена Владимировна, gutkevichelena@gmail.com}


For citation: Shtro R.S., Gutkevich E.V. Psychological risks of assisted reproductive technologies and especially the attitude of parents towards children born with ART (literature review). Siberian Herald of Psychiatry and Addiction Psychiatry. 2020; 2 (107): 51-59. https://doi.org/10.26617/1810-3111-2020-2(107)-51-59

\title{
Psychological risks of assisted reproductive technologies and especially the attitude of parents towards children born with ART (literature review)
}

\section{Shtro R.S., 1, 2 Gutkevich E.V.}

\author{
${ }^{1}$ National Research Tomsk State University \\ Lenin Avenue, 36, 634050, Tomsk, Russian Federation. \\ ${ }^{2}$ Mental Health Research Institute, Tomsk National Research Medical Center, Russian Academy of Sciences \\ Aleutskaya Street, 4, 634014, Tomsk, Russian Federation
}

\begin{abstract}
Assisted Reproductive Technology (ART) allows people with reproductive health problems to become pregnant, bear, and have a baby. Conception, pregnancy and birth of a child are accompanied by special psychological conditions and risks, and further interaction of parents and a child forms the vector of his/her mental development. The evidence determining the relevance of the topic is both an observed prevalence of reproductive health disorders and an increase in the use of assisted reproductive technologies, as well as real psychological risks for parents using ART, in particular, in vitro fertilization (IVF), and for their children. Objective: based on the materials of domestic and foreign literature, to study psychological characteristics of the use of assisted reproductive technologies in families with infertility and to systematize data on the presence or absence of associations between their use and the attitude of parents towards their children conceived, born and given birth through these technologies. Material and Methods: articles published from 1990 to 2019 in Russian and English are considered. The search has been carried out from February to April 2020, using the online databases Google Scholar, eLibrary and ScienceDirect, the preference is given to full-text articles of recent years, published in peer-reviewed scientific journals. Search queries include key phrases: "psychological aspects of ART/IVF", "parent-child relationships", "marital relations" and others, as well as their analogues in English. In previous studies of psychological well-being of parents using assisted reproductive technologies, the psychological risks of ART have been rarely considered for the formation of parent-child relationships, including the specifics of parent-child relationships; as well as psychological traits and dynamics of the attitude of parents towards their children, depending on the age of the child given birth using IVF. Study of this problem in the future will require additional data regarding equally the maternal and child-father relationships, as well as their dynamics and internal characteristics of various areas of emotional and personal communication between parents and children of different age in families using assisted reproductive technologies.
\end{abstract}

Keywords: psychological risks, infertility, assisted reproductive technologies (ART), in vitro fertilization (IVF), marital and family relationships, psychological aspects of parent-child relationships.

Received April 20.2020

Accepted June 01.2020

Shtro Roman S. - student of the Faculty of Psychology, National Research Tomsk State University, Tomsk, Russian Federation.

Gutkevich Elena V. - MD, lead researcher, Endogenous Disorders Department, Mental Health Research Institute, Tomsk National Research Medical Center, Russian Academy of Sciences, Tomsk, Russian Federation. Researcher ID O-1311-2014. Author ID Scopus 7801397871. ORCID 0000-0001-7416-7784. Author ID RSCI 165414. SPIN-code RSCI 6427-9007.

Gutkevich Elena V., gutkevichelena@gmail.com 